\title{
Middle East respiratory syndrome coronavirus (MERS- $\mathrm{CoV}$ ) in dromedary camels, Oman, 2013
}

N Nowotny (Norbert.Nowotny@vetmeduni.ac.at) ${ }^{1,2}$, J Kolodziejek ${ }^{1}$

1. Viral Zoonoses, Emerging and Vector-Borne Infections Group, Institute of Virology, University of Veterinary Medicine Vienna, Vienna, Austria

2. Department of Microbiology and Immunology, College of Medicine and Health Sciences, Sultan Qaboos University, Muscat, Oman

Nowotny N, Kolodziejek J. Middle East respiratory syndrome coronavirus (MERS-CoV) in dromedary camels, Oman, 2013. Euro Surveill. 2014;19(16):pii=20781. Available online: http://www.eurosurveillance.org/ViewArticle.aspx?Articleld=20781

A countrywide survey in Oman revealed Middle East respiratory syndrome coronavirus (MERS-CoV) nucleic acid in five of 76 dromedary camels. Camel-derived MERS-CoV sequences (3,754 nucleotides assembled from partial sequences of the open reading frame (ORF)1a, spike, and ORF4b genes) from Oman and Qatar were slightly different from each other, but closely related to human MERS-CoV sequences from the same geographical areas, suggesting local zoonotic transmission. High viral loads in nasal and conjunctival swabs suggest possible transmission by the respiratory route.

\section{Background}

In June 2012 a novel betacoronavirus, subsequently named Middle East respiratory syndrome coronavirus (MERS-CoV), was isolated from a patient with fever and respiratory symptoms who had been admitted to a hospital in Jeddah, Saudi Arabia [1]. As of 22 April 2014, the number of reported laboratory-confirmed cases of MERS-CoV worldwide amounts to 333, with 107 deaths [2]. To date, only fifteen cases, including ten in Europe, have been reported outside of the Middle East [2] and the vast majority of cases were reported from Saudi Arabia. Thus, it seems that the virus may originate from the Arabian Peninsula. Family, healthcare associated and community case clusters of MERS-CoV infections have been reported (e.g. [3]).

Besides limited human-to-human transmission, however, epidemiological data point towards an animal reservoir of MERS-CoV. First evidence of such a reservoir host was provided when all 50 investigated sera of dromedary camels (Camelus dromedaries) from Oman exhibited high-titre neutralising antibodies against MERS-CoV [4]. This observation has meanwhile been confirmed by several studies from other countries on the Arabian Peninsula and beyond (e.g. Egypt [5]). Other important farm animals on the Arabian Peninsula such as cattle, goats, sheep and chickens were also investigated but were found negative $[6,7]$. Although different species of bats carry a variety of coronaviruses and have been suggested as the most likely primary animal reservoir for MERS-CoV, so far only a short (190 nucleotides (nt)) sequence in a conserved region of the MERSCoV genome was amplified from a faecal pellet of a bat (Taphozous perforates) in Saudi Arabia [8]. The present study was initiated in order to identify the virus in camels and to compare it genetically with human-derived MERS-CoV.

\section{Sample collection}

In December 2013, nasal and conjunctival swabs were taken from 76 dromedary camels of different age, breed and sex from all over Oman. All swabs were taken in duplicates, one stored in virus isolation medium and the other in the virus-inactivating buffer DNA/RNA Shield (Zymo Research, Irvine, USA). The former samples were frozen at $-80^{\circ} \mathrm{C}$ at the Veterinary Research Center of the Ministry of Agriculture and Fisheries, Oman, for later use, while the latter samples were shipped to the University of Veterinary Medicine Vienna for analysis. DNA/RNA Shield effectively lyses cells and inactivates nucleases and infectious agents. In addition it ensures nucleic acid stability during sample storage and transport at ambient temperatures.

\section{Nucleic acid extraction and polymerase} chain reactions

RNA was extracted employing a Quick-RNA MiniPrep Kit (Zymo Research, Irvine, USA), following the manufacturer's instructions.

For screening, two published MERS-CoV reverse transcription-quantitative polymerase chain reaction (RT-qPCR) assays were performed, one in the open reading frame (ORF)1a gene region [9] and the other in the ORF1b gene region [10], and both were optimised for SuperScript III Platinium One-Step RT-qPCR System (Invitrogen/LifeTechnologies, CA, USA). For confirmation, the positive samples were retested by reverse transcription-polymerase chain reactions (RT-PCRs) in the RNA-dependent RNA polymerase (RdRp)- and nucleocapsid $(\mathrm{N})$ gene regions [9] as well as in the spike 
Primers used for amplification of Middle East respiratory syndrome coronavirus genetic sequences and subsequent phylogenetic analysis, 2013

\begin{tabular}{|c|c|c|}
\hline Primer name, position ${ }^{a}$ and sequence & Target sequence & Expected size \\
\hline $\begin{array}{l}\text { MERS_1767_F: 5'-CTCGCAATTCTCTCTGGAAC-3' } \\
\text { MERS_2615_R: 5'-GTCAGTAGGTTGGAGCAGTC-3' }\end{array}$ & ORF1a & $848 \mathrm{nt}$ \\
\hline $\begin{array}{l}\text { MERS_11419_F: 5'-CAA GCC CCA TTG CCT ATC TG-3' } \\
\text { MERS_12064_R: 5'-GCT TGA AGT ACG CTA GGA GTG-3' }\end{array}$ & ORF1a & $645 \mathrm{nt}$ \\
\hline $\begin{array}{l}\text { MERS_22074_F: 5'-CGTAATGCCAGTCTGAACTC-3' } \\
\text { MERS_23127_R: 5'-CAGGGTGAGTATTGATTAGCG-3' }\end{array}$ & Spike & $958 \mathrm{nt}$ \\
\hline $\begin{array}{l}\text { MERS_24156_F: 5'-GCTGATCCTGGTTATATGCAAGG-3' } \\
\text { MERS_24903_R: 5'-CAACCTCAATGTGGTTGCTAGG-3' }\end{array}$ & Spike & $747 \mathrm{nt}$ \\
\hline $\begin{array}{l}\text { MERS_26042_F: 5'-CTT TGG CCA AAC AGG ACG CA-3' } \\
\text { MERS_26856_R: 5'-GAC GCC GAG AAA GCC ATA GTT C-3' }\end{array}$ & $\mathrm{ORF}_{4} \mathrm{~b}$ & $814 \mathrm{nt}$ \\
\hline
\end{tabular}

F: forward; MERS: Middle East respiratory syndrome; nt: nucleotide; ORF: open reading frame; R: reverse.

${ }^{a}$ Referring to the sequence of the MERS-coronavirus strain HCoV-EMC/2012 with the GenBank accession number: JX869059.

gene region [11] and by five other RT-PCRs designed for subsequent phylogenetic analysis (Table). The MERSCoV genome organisation and the location of the five RT-PCR amplicons used to generate a concatenated sequence for phylogenetic analysis are displayed in Figure 1.

All conventional RT-PCRs were conducted using OneStep RT-PCR kit (Qiagen, Hilden, Germany). Primer synthesis and sequencing in both directions were carried out by Microsynth (Balgach, Switzerland). The obtained sequences were verified by Basic Local Alignment Search Tool (BLAST) search, aligned using the ALIGN PLUS programme (Scientific \& Educational Software), and compiled to one concatenated sequence.

\section{Phylogenetic analysis}

For phylogenetic analysis, three camel-derived (from Oman, Qatar, and Egypt) and 33 human-derived MERSCoV sequences were included. A multiple sequence alignment was performed using BioEdit Sequence Alignment Editor version 7.0.9.0 and verified using the CLUSTAL_X programme (version 1.8). Phylogenetic neighbour-joining and maximum likelihood analyses were conducted with the help of the Molecular Evolutionary Genetics Analysis (MEGA) 5 programme [12]. The evolutionary distances were computed using the Kimura 2-parameter model. Bootstrap resampling analysis with 1,000 replicates was employed. The sequences used for phylogenetic analysis were deposited at GenBank under the accession numbers KJ573789-KJ573793 (MERSCoV sequences derived from camel Oman_30_2013), and KJ598493-KJ598496 and KF933384 (sequences derived from camel Qatar_1_2013).

\section{Results}

Nasal and conjunctival swabs of five of a total of 76 camels $(6.6 \%)$ proved positive in all applied RT-qPCR and RT-PCR assays. The cycle threshold $(\mathrm{Ct})$ values ranged from 15.74 to 36.29 . Concatenated sequences of a total of 3,754 nts were obtained from all five positive Omani camels by compiling the sequences derived from the five different PCR amplification products covering the ORF1a, spike and $\mathrm{ORF}_{4} \mathrm{~b}$ gene regions (Table, Figure 1). The five concatenated sequences derived from the Omani camels were $100 \%$ identical to each other, and they exhibited 99\% identity (differing in 12 of 3,754 nts) to the sequence derived from the Qatari

\section{FIGURE 1}

Genome organisation of Middle East respiratory syndrome coronavirus and location of five reverse transcriptionpolymerase chain reaction amplification products used to generate a concatenated sequence for phylogenetic analysis, 2013

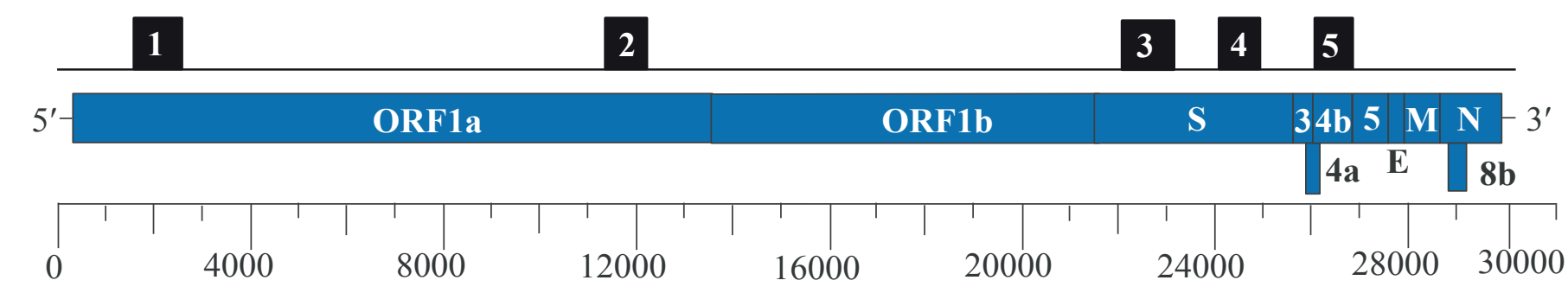




\section{FIGURE 2}

Phylogenetic analysis of three camel- and 33 human-derived Middle East respiratory syndrome coronavirus (MERS-CoV) nucleotide sequences, 2013

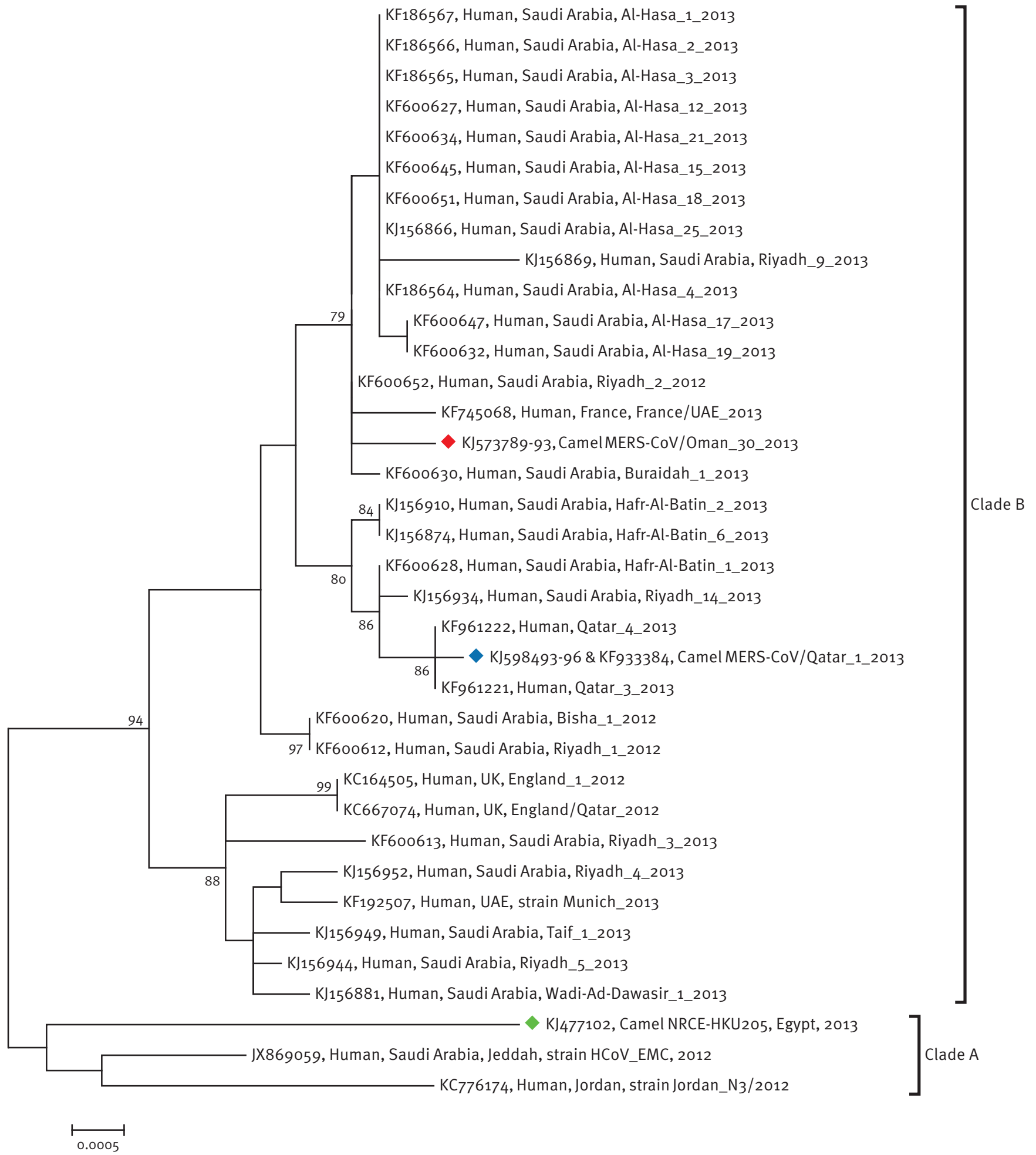

UAE: United Arab Emirates; UK: United Kingdom.

Each 3.754 nucleotide long sequence used to generate the tree was obtained from concatenating partial sequences of the open reading frame (ORF)1a, spike and ORF4b gene regions. Of note the different clustering of the camel-derived sequences originating from Oman (marked with a red diamond), Qatar (blue diamond) and Egypt (green diamond). The Qatari and Omani camel-derived MERS-CoV sequences cluster close to the human-derived sequences originating from the same areas. 
camel and also $99 \%$ identity (31 nts difference) to the known respective sequence derived from an Egyptian camel (Figure 2).

The phylogenetic analysis involving all three currently available camel-derived MERS-CoV sequences and all 33 available human-derived sequences is shown in Figure 2. It clearly indicates that the camel-derived MERS-CoV sequences are clustering independently from each other, but together with the human-derived MERS-CoV sequences from the same geographical areas. This could be demonstrated unambiguously for the Qatari samples (exhibiting only $1 \mathrm{nt}$ difference between the camel-derived and the human-derived MERS-CoV sequences), but also for the camel-derived Omani sequence, which is clustering close to a human MERS-CoV sequence from neighbouring United Arab Emirates (GenBank accession number: KF745068, with only 5 of 3,754 nts being different; no human Omani MERS-CoV sequences are available so far). For the Egyptian camel-derived MERS-CoV sequence [13] no human-derived sequence with as high relative sequence identity and/or from a respectively close geographical area has yet been detected.

\section{Discussion}

The results of our study and similar studies from Qatar [11] and Saudi Arabia [14-16] show a close genetic relationship between camel-derived and human-derived MERS-CoV from the same geographical areas, suggesting local zoonotic transmission. A proof of cross-species transmission of MERS-CoV from dromedary camels to humans was reported in a publication on human infection with MERS-CoV after exposure to infected camels in Saudi Arabia recently [15]. The authors concluded that camels may act as a direct source of human MERS$\mathrm{CoV}$ infection. For the implementation of effective, but reasonable precautions, however, further pieces of the puzzle must be put together: a Ct-value of 15.74 , corresponding to approximately 33 million RNA copies, in the nasal swab of one of the Omani camels (this study) indicates a very high virus load and the probability of the transmission of high numbers of virus particles by the nasal route. Data from another recent publication suggest that neither passively acquired maternal antibodies nor prior infections seem to lead to full immunity in the camels, and viral nucleic acid was detected in such animals, though at a significantly lower level [16]; these and other recent results, however, require confirmation.

Evidence was provided that MERS-CoV has been circulating in camels at least since 1992 [14], but probably much longer, while strains in humans emerged only recently [17]. Initially, dromedary camels have been considered possible intermediate hosts of MERS-CoV, while bats were suggested as primary reservoirs - in analogy to severe acute respiratory syndrome-coronavirus (SARS-CoV). According to our current knowledge, however, dromedary camels might even be considered the primary reservoir hosts of MERS-CoV. Comparative analyses of camel- and human-derived MERS-CoV sequences exhibit $>99 \%$ nt identities to each other with so far no striking genetic differences observed. Given the high prevalence of MERS-CoV-specific antibodies in dromedary camels $[4,5]$, infection of camels with this virus seems to be frequent on the Arabian Peninsula. This is in sharp contrast to the comparatively low number of recorded human infections. Since currently no other obvious explanation for this discrepancy exists, it is postulated that a high infectious dose through very close contact between an infected camel and a human being is required for initiation of human MERS-CoV infection by camels. Younger camels may play a particular role in zoonotic transmission since they seem to be more frequently infected and seem to shed more virus than older ones [14-16]. Although the respiratory route is, in our opinion, the most likely route of transmission, a recent paper demonstrated that MERS-CoV survived in raw camel milk slightly longer than in milk of other species [18], suggesting further investigations on a possible food-borne route of transmission. Also, it is not clear yet whether the course of MERS-CoV infection in camels is generally asymptomatic or associated with mild respiratory symptoms, as suggested recently [16].

\section{Conclusions}

Phylogenetic analysis and high MERS-CoV viral load in nasal swabs of dromedary camels suggest local zoonotic transmission through the respiratory route.

\section{Acknowledgments}

This study was funded by the University of Veterinary Medicine Vienna. We wish to thank Drs. Abdulmajeed AlRawahi, Muhammad Hammad Hussain and Mohammed Body from the Veterinary Research Center, Ministry of Agriculture \& Fisheries, Sultanate of Oman, as well as Omani field veterinarians for sample obtainment, Dr. Idris Saleh Al-Abaidani from the Department of Communicable Disease Surveillance and Control, Ministry of Health, Muscat, Sultanate of Oman, for information on the human Omani MERS-CoV cases, and Drs. Khuloud Al Maamari and Fatma Ba'Alawi from the Department of Microbiology \& Immunology, Sultan Qaboos University Hospital, as well as Dr. Karin Pachler and Michael Kolodziejek from the Institute of Virology, University of Veterinary Medicine Vienna, for their technical help. We would also like to thank Prof. Karin Möstl from the Institute of Virology, University of Veterinary Medicine Vienna, for useful information about a new virus inactivation method. We are especially thankful to Drs. Bart L. Haagmans and V. Stalin Raj from the Department of Viroscience, Erasmus Medical Center, Rotterdam, The Netherlands, for providing primer sequences, MERS-CoV sequences from the Qatari camel, and Figure 1.

Conflict of interest

None declared.

Authors' contributions 
NN designed, coordinated and supervised the study and wrote the manuscript; JK performed laboratory testing, analysed data, read and revised manuscript.

\section{References}

1. Zaki AM, van Boheemen S, Bestebroer TM, Osterhaus AD, Fouchier RA. Isolation of a novel coronavirus from a man with pneumonia in Saudi Arabia. N Engl J Med. 2012;367(19):181420.

http://dx.doi.org/10.1056/NEJMoa1211721

2. European Centre for Disease Prevention and Control (ECDC). Epidemiological update: First imported case of MERS-CoV in Greece. Stockholm: ECDC. [Accessed 22 Apr 2014]. Available from: http://www.ecdc.europa. eu/en/press/news/_layouts/forms/News_DispForm. aspx? List=8db7286c-fe2d-476c-9133-18ff4cb1b568\&ID=989

3. Assiri A, McGeer A, Perl TM, Price CS, Al Rabeeah AA, Cummings DA, et al. Hospital outbreak of Middle East respiratory syndrome coronavirus. N Engl J Med. 2013;369(5):407-16. http://dx.doi.org/10.1056/NEJMoa1306742

4. Reusken CB, Haagmans BL, Müller MA, Gutierrez C, Godeke GJ, Meyer B, et al. Middle East respiratory syndrome coronavirus neutralising serum antibodies in dromedary camels: a comparative serological study. Lancet Infect Dis. 2013;13(10):859-66. http://dx.doi.org/10.1016/S1473-3099(13)70164-6

5. Perera RA, Wang P, Gomaa MR, El-Shesheny R, Kandeil A, Bagato O, et al. Seroepidemiology for MERS coronavirus using microneutralisation and pseudoparticle virus neutralisation assays reveal a high prevalence of antibody in dromedary camels in Egypt, June 2013. Euro Surveill. 2013;18(36). pii: 20574 .

6. Hemida MG, Perera RA, Wang P, Alhammadi MA, Siu LY, Li M, et al. Middle East Respiratory Syndrome (MERS) coronavirus seroprevalence in domestic livestock in Saudi Arabia, 2010 to 2013. Euro Surveill. 2013;18(50). pii: 20659.

7. Reusken CB, Ababneh M, Raj VS, Meyer B, Eljarah A, Abutarbush S, et al. Middle East Respiratory Syndrome coronavirus (MERS-CoV) serology in major livestock species in an affected region in Jordan, June to September 2013. Euro Surveill. 2013;18(50). pii: 20662.

8. Memish ZA, Mishra N, Olival KJ, Fagbo SF, Kapoor V, Epstein JH, et al. Middle East respiratory syndrome coronavirus in bats, Saudi Arabia. Emerg Infect Dis. 2013;19(11):1819-23. http://dx.doi.org/10.3201/eid1911.131172

9. Corman VM, Müller MA, Costabel U, Timm J, Binger T, Meyer $B$, et al. Assays for laboratory confirmation of novel human coronavirus (hCoV-EMC) infections. Euro Surveill. 2012;17(49). pii: 20334 .

10. Corman VM, Eckerle I, Bleicker T, Zaki A, Landt O, Eschbach Bludau M, et al. Detection of a novel human coronavirus by real-time reverse-transcription polymerase chain reaction. Euro Surveill. 2012;17(39). pii: 20285. Erratum in: Euro Surveill. 2012;17(40). pii: 20288.

11. Haagmans BL, Al Dhahiry SH, Reusken CB, Raj VS, Galiano M, Myers R, et al. Middle East respiratory syndrome coronavirus in dromedary camels: an outbreak investigation. Lancet Infect Dis. 2014;14(2):140-5. http://dx.doi.org/10.1016/S1473-3099(13)70690-X

12. Tamura K, Peterson D, Peterson N, Stecher G, Nei M, Kumar S. MEGA5: Molecular Evolutionary Genetics Analysis using maximum likelihood, evolutionary distance, and maximum parsimony methods. Mol Biol Evol. 2011;28(10):2731-9.http:// dx.doi.org/10.1093/molbev/msr121

13. Chu DKW, Poon LLM, Gomaa MM, Shehata MM, Perera RAPM, Zeid DA, et al. MERS coronaviruses in dromedary camels, Egypt. Emerg Infect Dis. 2014;20(6). [Epub ahead of print]. http://dx.doi.org/10.3201/eid2006.140299

14. Alagaili AN, Briese T, Mishra N, Kapoor V, Sameroff SC, de Wit E, et al. Middle East respiratory syndrome coronavirus infection in dromedary camels in Saudi Arabia. MBio. 2014;5(2):e00884-14. http://dx.doi.org/10.1128/mBio.01002-14

15. Memish ZA, Cotten M, Meyer B, Watson SJ, Alsahafi AJ, Al Rabeeah AA, et al. Human infection with MERS coronavirus after exposure to infected camels, Saudi Arabia, 2013. Emerg Infect Dis. 2014:20(6). [Epub ahead of print]. http://dx.doi.org/10.3201/eid2006.140402

16. Hemida MG, Chu DKW, Poon LLM,. Perera RAPM, Alhammadi MA, Ng H-Y, et al. MERS coronavirus in dromedary camel herd, Saudi Arabia. Emerg Infect Dis. 2014:20(7). [Epub ahead of print].

http://dx.doi.org/10.3201/eid2007.140571

17. Cotten M, Watson SJ, Kellam P, Al-Rabeeah AA, Makhdoom HQ, Assiri A, et al. Transmission and evolution of the Middle East respiratory syndrome coronavirus in Saudi Arabia: a descriptive genomic study. Lancet. 2013;382(9909):1993-2002. http://dx.doi.org/10.1016/S0140-6736(13)61887-5

18. van Doremalen N, Bushmaker T, Karesh WB, Munster VJ. Stability of Middle East respiratory syndrome coronavirus in milk [letter]. Emerg Infect Dis. 2014;20(7). [Epub ahead of print].

http://dx.doi.org/10.3201/eid2007.140500 